\title{
Liebe Leserinnen und Leser, liebe Mitglieder der SGG
}

Sie halten heute die Erstausgabe einer brandneuen Fachzeitschrift in der Hand:

\section{Angewandte GERONTOLOGIE!}

Die Angewandte GERONTOLOGIE ist nicht nur eine neue Fachzeitschrift, sondern auch die neue Mitgliederzeitschrift der Schweizerischen Gesellschaft für Gerontologie (SGG)!

Die Angewandte GERONTOLOGIE ist sichtbarer Ausdruck der Bemühungen der SGG, die Professionalisierung, Vernetzung und die multidisziplinäre Zusammenarbeit im Arbeitsfeld Alter(n) weiter voranzutreiben: Mit dieser Zeitschrift stellt sie der wachsenden Gemeinschaft der praktisch tätigen Gerontologinnen und Gerontologen ein angemessenes Medium zur Verfügung. Ganz im Sinne der SGG greift die Angewandte GERONTOLOGIE brisante Themen rund ums Alter(n) auf und informiert über aktuelle Entwicklungen und Meinungen im Feld, um so vertiefte Fachdiskussionen anzuregen.

Angesprochen werden sollen mit der neuen Fachzeitschrift Fachleute im Arbeitsfeld Alter(n), Studierende sowie weitere Berufsleute, die zwar nicht explizit im Altersbereich tätig sind, jedoch in ihrem Berufsalltag mit Fragen rund ums Alter(n) konfrontiert werden und neue Impulse für ihren Tätigkeitsbereich suchen.

\section{Warum braucht es die Angewandte GERONTOLOGIE?}

In unserer Gesellschaft des langen Lebens benötigt es mehr denn je spezifische gerontologische Erkenntnisse. Angesichts der demografischen Entwicklung sind dabei nicht nur neue Forschungsergebnisse gefragt, sondern vor allem auch deren Umsetzung in die Praxis. In der Schweiz besteht aktuell eine Lücke bei der angemessenen Vermittlung der vielen bereits vorhandenen Informationen und Erkenntnisse in die Praxis der angewandten Gerontologie. Die neue Fachzeitschrift möchte genau diese Lücke schliessen und ein breit abgestütztes Forum bieten, das gezielt die Anwendung gerontologischer Erkenntnisse und den Transfer zwischen Theorie und Praxis in den Blick nimmt.

Als Mitgliederorgan der SGG ersetzt die neue Fachzeitschrift die bisherige Gerontologie Information und ist als solche für SGG-Mitglieder im jährlichen Mitgliederbeitrag inbegriffen. Nichtmitglieder können sie im freien Abonnement oder als Einzelheft beziehen.

Die Angewandte GERONTOLOGIE erscheint zukünftig viermal jährlich mit einem Themenschwerpunkt. Die vorliegende Erstausgabe widmet sich dem Thema «Alter und Technik», dem diesjährigen Schwerpunkt des SGG-Kongresses. Verschiedene Referierende werden Sie denn auch mit Beiträgen in diesem Heft wiederfinden.
Angewandte GERONTOLOGIE möchte schweizweit breit informieren. Daher werden alle Artikel jeweils in der Sprache der Autorinnen und Autoren publiziert (Deutsch, Französisch oder Italienisch). Zusätzlich wird für jeden Beitrag eine kurze Zusammenfassung jeweils auf Deutsch respektive Französisch übersetzt und abgedruckt. So können Sie sich kurz über den Inhalt der Artikel informieren, um dann zu entscheiden, ob Sie die Vollversion des Artikels in der jeweils gewünschten Sprache online nachlesen möchten. Online werden nämlich alle Beiträge in Originallänge sowohl auf Deutsch wie auf Französisch zur Verfügung gestellt. Dieser Service ist im Abonnement und im Kauf eines Einzelheftes inbegriffen. Auf diese Weise möchte die SGG dazu beitragen, den Austausch der angewandt tätigen Gerontologinnen und Gerontologen schweizweit zu fördern.

Ich freue mich ganz ausserordentlich, dass es mit der neuen Fachzeitschrift Angewandte GERONTOLOGIE gelungen ist, einen Meilenstein für die angewandte Gerontologie in der Schweiz zu realisieren. Ganz herzlich danken möchte ich an dieser Stelle sowohl dem Vorstand der SGG für die im Vorfeld wichtigen Diskussionen, dem Hogrefe Verlag in Bern für die wertvolle Unterstützung im Erarbeitungsprozess, dem Redaktionsteam (Mitglieder siehe Impressum) für die engagierte und kompetente Mitarbeit sowie allen unseren Autorinnen und Autoren, die massgeblich dazu beigetragen haben, dass wir Ihnen heute die Erstausgabe Angewandte GERONTOLOGIE präsentieren können!

Ich hoffe sehr, dass Ihnen das Lesen Freude bereitet und Anregung bietet. Gerne nehme ich jederzeit Ihre Rückmeldungen und Anregungen für zukünftige Ausgaben entgegen.

Der Schwerpunkt der zweiten Ausgabe 2016 wird sich mit dem «Berufsbild Gerontologin/Gerontologe» auseinandersetzen.

Vielleicht darf ich ja auch Sie demnächst mit einem eigenen Beitrag als Autorin oder Autor begrüssen!
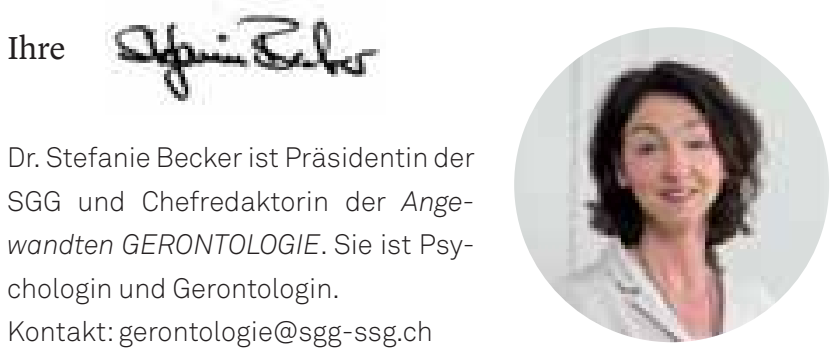